\title{
First-principles studies on organic electronic materials
}

L. Tsetseris ${ }^{1,2}$ and S. T. Pantelides ${ }^{2,3 a}$

${ }^{1}$ Department of Physics, Aristotle University of Thessaloniki, GR-54124 Thessaloniki, Greece

2 Department of Physics and Astronomy, Vanderbilt University, Nashville, Tennessee 37235, USA

${ }^{3}$ Oak Ridge National Laboratory, Oak Ridge, Tennessee 37831, USA

Received: date / Revised version: date

Abstract. The elucidation of physical properties of organic materials is important for further optimization of related electronic and optoelectronic devices. Here we review briefly various first-principles computational tools for the modeling of these materials by investigating key structural, electronic, and chemical properties of prototype organic semiconductors. In particular, we discuss the site-selectivity for band formation in pentacene and rubrene, hydrogenation and transformations of metal-free phthalocyanines, and the bonding topology in a hybrid organic-inorganic system.

PACS. 61.66.Hq Structure of specific crystalline solids - Organic compounds - 71.20.Rv Electron density of states and band structure of crystalline solids - Polymers and organic compounds - 81.07.Pr Nanoscale materials and structures: fabrication and characterization - Organic-inorganic hybrid nanostructures

\section{Introduction}

Electronic devices based on organic oligomers and polymers have attracted a resurgent interest in recent years.[16] Low cost of production and flexibility provide them with distinct advantages when compared to traditional electronic systems made of inorganic materials like silicon.

\footnotetext{
a Correspoonding author: L. Tsetseris, phone number: +30-2310-998039, fax number: +30-2310-998039, e-mail: tsetser@auth.gr
}

The continuous expansion in usage of organic electronic materials requires, however, the resolution of several issues that relate to key performance indicators of respective devices and important properties, like carrier mobilities or chemical stability.

Theoretical modeling is an integral part of the development and optimization of organic electronics. Given that the corresponding physical systems typically span several different scales, both spatially and temporally, vari- 
ous techniques are employed to study the properties of organic materials and devices. Approaches range from device modeling based on continuity equations and electrostatics, to Monte-Carlo simulations of carrier and energy transfer in meso-scales, [7] to atomic-scale modeling with firstprinciples (also known as ab initio) methods.[3,6] With the increase of available computational power, the role of the latter quantum-mechanical approaches in the modeling of organic electronic materials has been steadily expanding.

In this article we study a number of important organicbased systems and demonstrate how first-principles calculations can elucidate the atomic-scale details of various mechanisms that control the operation of related electronic devices. We show that the central rings in pentacene and rubrene have an enhanced contribution in the formation of the valence and conduction bands, and, by the same token, they play a crucial role for carrier transport, especially in the case of rubrene. We describe the hydrogenation and transformations of isomers for metalfree phthalocyanines $(\mathrm{Pc})$ and possibilities of physisorbed and chemisorbed binding of a $\mathrm{Si}$ nanocluster on a PPV [poly(paraphenylenevinylene)] chain. We conclude with a short discussion of carrier hopping in organic materials as described by the Marcus and Mills-Abrahams models.

\section{Methodology}

The results we present below were obtained with parameter- sity of states (DOS) of a pentacene polytype [18] based free, first-principles density-functional theory (DFT) calculations. The exchange and correlation (xc) energies of the many-electron problem were described with local-density phase comprises two Pn molecules as shown in Fig. 2(a) approximation (LDA)[8] or generalized gradient corrected (GGA)[9] functionals. Plane waves were used as basis functions and the interaction of valence electrons with ionic cores was described with ultrasoft pseudopotentials (US$\mathrm{PP})[10]$ or projector augmented waves (PAW),[11] as implemented in the code VASP.[12] Isolated defects were modeled with sufficiently large supercells. Convergence of results was checked with respect to relevant quantities, namely the size of supercells, and the number of kpoints for Brillouin zone sampling.[13] Reaction and transformation barriers were obtained with the elastic band method [14] based on experience with various systems.[15, 16] Combining barriers with typical attempt frequencies in the Arrhenious expression for event rates allows for an estimate of the time scale of processes at certain temperatures. For example, when a process has a barrier of 0.5 $\mathrm{eV}, 0.9 \mathrm{eV}$, or $1.5 \mathrm{eV}$ and the attempt frequency is $10^{13}$ $\mathrm{Hz}$, then the respective time scale for activation at room temperature is about $50 \mu \mathrm{sec}, 400 \mathrm{sec}$, and $10^{13} \mathrm{sec}$, respectively.

\section{Results and discussion}

Pentacene $(\mathrm{Pn})$ is one of the most important organic semiconductors $[1,2,6]$ because of its relatively high carrier mobility and the ability of Pn films to grow on a variety of substrates.[17] In Fig. 1 we show the electronic denon DFT-LDA calculations and $k$-point sampling with the tetrahedron method.[19] The unit cell of the crystalline 


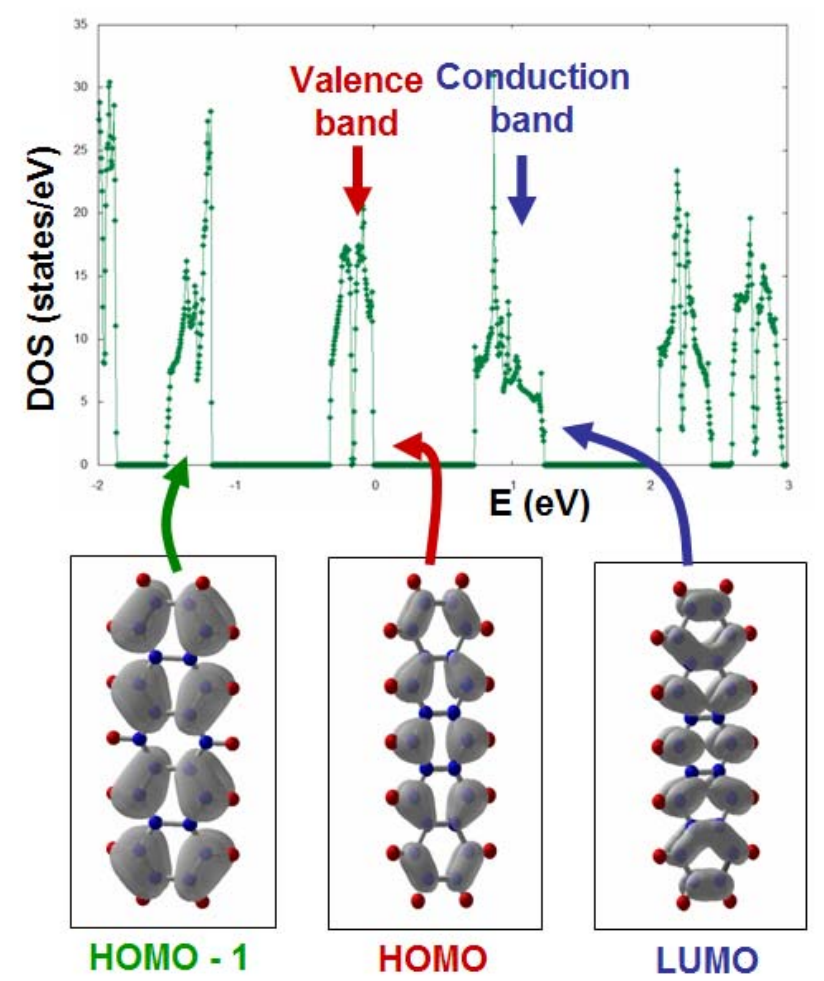

Fig. 1. Band formation in a pentacene crystal: (top) electronic density of states of pentacene, and (bottom) amplitudes of the respective highest occupied (HOMO) and lowest unoccupied (LUMO) orbitals of pentacene. The orbital below the HOMO (HOMO-1) is also shown.

and when it is repeated periodically it forms a herringbone pattern that is typical to many organic semiconductors. We should note that, due to a well known limitation of LDA and GGA DFT functionals, the calculated band gap of Fig. 1 is significantly smaller than the experimental value [20] of $2.2 \mathrm{eV}$. This shortcoming can be corrected, at least partially, with the employment of more elaborate theoretical approaches such as the so-called GW method.[21]

In Fig. 1 we show the amplitudes of the highest occupied (HOMO) and lowest unoccupied (LUMO) orbitals of a pentacene molecule. Though the differences are not pronounced there is a slight preference of both the HOMO and the LUMO for the area around the central carbon rings, which in turn results in a slightly larger contribution of the central ring in the formation of the valence and conduction bands of Pn. The effect is demonstrated through the projected DOS (PDOS) of Pn shown in Fig. 2(b).

The site-selectivity in the formation of bands is much more pronounced in the case of rubrene, which is currently the record-holder organic semiconductor in terms of carrier mobilities.[4,22] A rubrene molecule comprises a tetracene backbone and four side phenyl groups. The PDOS (Fig. 3) of crystalline rubrene [23] shows that the contribution of the tetracene backbone, and especially its central rings, is significantly larger than that of the side phenyl groups of the molecule. Similar to pentacene, the DOS site-selectivity of rubrene can be traced also in the form of the corresponding HOMO and LUMOs. Actually, the rubrene HOMO and LUMO, which are depicted in Ref. [24], have a very small weight in the phenyl groups, a fact the underlies the miniscule role of these parts in the formation of the valence and conduction bands.

The results of Figs. 2-3 have implications also for the effect of impurities in these organic semiconductors. In particular, based on the rubrene PDOS profile of Fig. 3(b) it can be expected that any disruption of the $\pi$ - $\pi$ stacking between neighboring molecules in a rubrene crystal will lead to significant changes in the valence and conduction bands. Indeed, such large changes are reported in Ref. [24] for oxygen and hydrogen impurities that chemisorb on the 


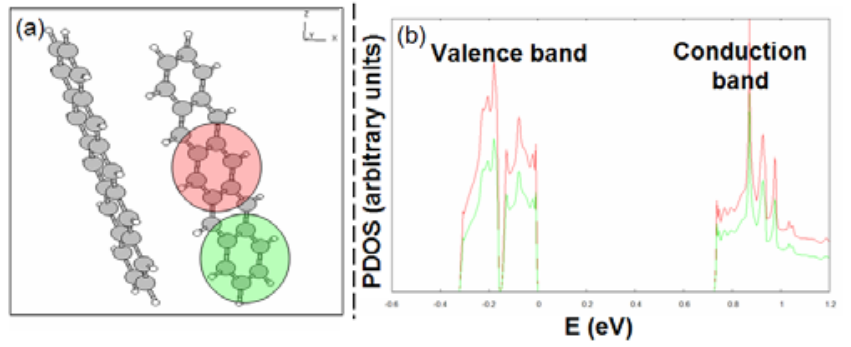

Fig. 2. Contribution of the encircled $\mathrm{C}$ rings of (a) in the electronic density of states (b) of pentacene.

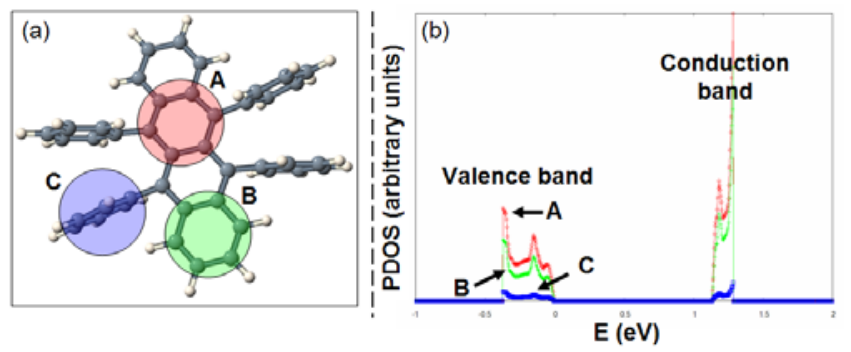

Fig. 3. Contribution of the encircled $\mathrm{C}$ rings of (a) in the electronic density of states (b) of rubrene. The tetracene backbone of rubrene has an enhanced role in the formation of the valence and conductions bands.

tetracene backbone of rubrene, and, in cases, produce energy levels in the band gap of the material. Oxygen and hydrogen species produce also levels in the energy band gap of pentacene. $[25,26]$ These theoretical findings are consistent with pertinent experimental observations about oxygen and hydrogen-related carrier traps in rubrene $[27,28]$ and pentacene.[29]

Hybrid systems with mixtures of organic and inorganic materials are currently being investigated intensively in pursuit of the optimal balance between the normally superior physical properties of inorganic systems and the low cost and flexibility of their organic counterparts. In addition, in many applications there are interfaces between organic and inorganic species, for example between carbon nanotubes and proximal dielectrics.[30] One of the most important issues at these boundaries is the nature of bonding between the materials, and, especially, whether physisorption or chemisorption occurs. The availability of reactive sites can, in fact, facilitate the selective docking of organic molecules like pentacene [31] or other oligoacenes [32] on important substrates such as silicon.

A state-of-the-art approach to characterize the chemical bonding between species is based on the so-called electron localization function (ELF).[33] ELF values range from 0 to 1 and the two extremes describe very weak and very strong repulsion, respectively, between two electrons of the same spin. In this context, a covalent bond typically appears in ELF studies as isolated isosurfaces between neighboring atoms and for large ELF values $f$ (normally for $f \geq 0.7$ ). Fig. 4 depicts two configurations of a $\mathrm{Si}$ nano-cluster with 27 atoms $[34,35]$ next to a PPV chain. At the top we show a stable structure with the cluster in the vicinity of the polymer; the binding energy of about $0.4 \mathrm{eV}$ is considerable, although the ELF contour plots do not reveal any so-called disynaptic basin that would indicate the formation of a covalent bond. In contrast, in the lower structure of Fig. 4 the cluster chemisorbs on an aliphatic $\mathrm{C}$ atom of the PPV chain and a bond is formed as demonstrated in the respective ELF plot. The competition between the formation of the attractive covalent link and the repulsion related to the disruption of the stable $s p^{2}$ PPV structure results in this case to a smaller binding energy of $0.2 \mathrm{eV}$. 


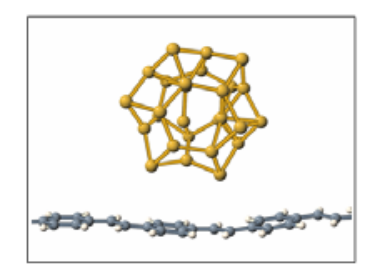

Binding energy $\sim 0.4 \mathrm{eV}$

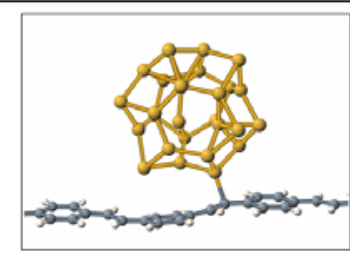

Binding energy $\sim 0.2 \mathrm{eV}$

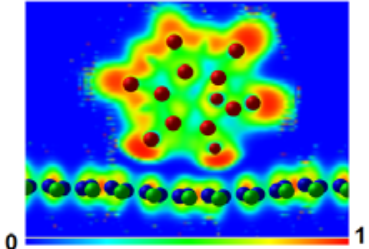

ELF

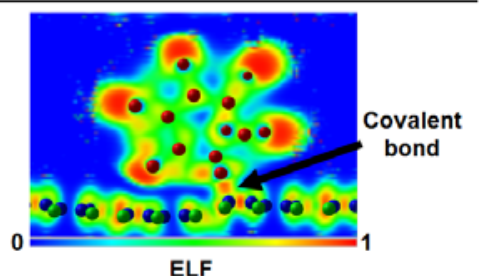

ELF

Fig. 4. Stable configurations of the hybrid system of a Si cluster with 27 atoms and a PPV chain. The images on the right are contour plots of the electron localization function (ELF), showing the formation of a covalent bond between the Si cluster and the PPV chain in the second case.

We now switch to phthalocyanines, another class of prototype organic materials $[36,37]$ which are used in various important applications, for example photovoltaics or cancer treatment. Here we discuss how first-principles calculations (DFT-GGA, unless stated otherwise) can describe the kinetics of reactions and transformations of metal-free phthalocyanines (Pc).

Metal-free Pc's typically contain two $\mathrm{H}$ atoms [38] at their core ( $\mathrm{H} 2 \mathrm{Pc})$, and trans and cis isomer configurations are possible with respect to the position of these $\mathrm{H}$ species. Figs. 5(a) and (c) depict these trans and cis isomers, and the transition state (TS) for the inter-isomer transformation is shown in Fig. 5(b). The DFT-GGA reaction energy $(\Delta E)$ and barrier $\left(E_{a}\right)$ are equal to $0.34 \mathrm{eV}$ and $0.49 \mathrm{eV}$, respectively, while the corresponding DFT-LDA values are $0.28 \mathrm{eV}$ and $0.36 \mathrm{eV}$. The trans isomer is more stable, as in previous studies [39] that reported $\Delta E$ and $E_{a}$ values
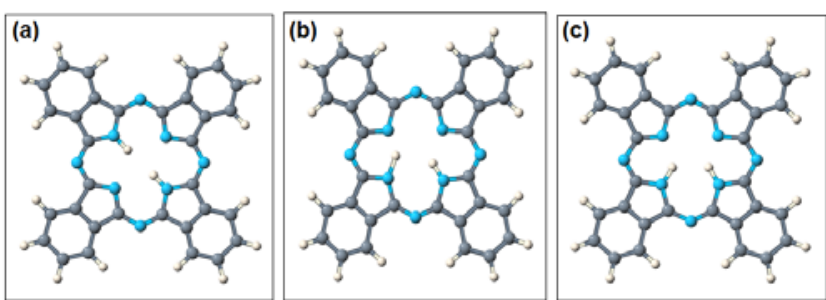

Fig. 5. Transformation of metal-free phthalocyanine (Pc) with $2 \mathrm{H}$ atoms at the Pc core: (a) and (c) are inital and final configurations, (b) is the transition state. The DFT-GGA energies of (b) and (c) are larger than that of (a) by $0.49 \mathrm{eV}$ and 0.34 $\mathrm{eV}$, respectively.

of $0.38 \mathrm{eV}$ and $0.66 \mathrm{eV}$ based on the B3LYP xc-functional. The transformation of Fig. 5 can be viewed also one of two equivalent steps that can lead to rotation of the inner $\mathrm{H}$ pair between passivated and unpassivated core $\mathrm{N}$ atoms. The relatively low $E_{a}$ values indicate that at room temperature the inner $\mathrm{H}$ pair rotates rapidly, a fact that is consistent with experimental data [39] that assign a half $\mathrm{H}$-occupancy for the core $\mathrm{N}$ atoms of H2Pc. We studied also the possibility of a single-step rotation of the $\mathrm{H}$ pair, whence the core $\mathrm{H}$ atoms migrate simultaneously to neighboring $\mathrm{N}$ sites. The elastic-band relaxation of this reaction pathway led to a sequence of steps of the form of Fig. 5 .

Deviations from the $\mathrm{H} 2 \mathrm{Pc}$ stoichiometry in terms of core $\mathrm{H}$ content may appear either during $\mathrm{Pc}$ growth or under long term operation of Pc-based devices, given that $\mathrm{H}$ species are often present and highly mobile in a variety of electronic systems. We investigated the propensity of Pc to uptake hydrogen from an $\mathrm{H}_{2}$ ambient. Starting with $\mathrm{H} 0 \mathrm{Pc}$ (no $\mathrm{H}$ at the core) there is a significant gain of 2.62 $\mathrm{eV}$ in energy for the uptake of one core $\mathrm{H}$ atom. Adding a 
second $\mathrm{H}$ atom leads to a further decrease of $2.16 \mathrm{eV}$. The next hydrogenation step (formation of H3Pc) is, however, endothermic with an energy penalty of $0.35 \mathrm{eV}$. Addition of a fourth $\mathrm{H}$ atom is accompanied with a slight energy increase of $0.21 \mathrm{eV}$. It should be noted that it is energetically favorable for two neighboring $\mathrm{H} 2 \mathrm{Pc}$ and $\mathrm{H} 0 \mathrm{Pc}$ species to transform to two H1Pc molecules, whereas two neighboring $\mathrm{H} 3 \mathrm{Pc}$ entities are favored to create a $\mathrm{H} 2 \mathrm{Pc}$ and a H4Pc molecule.

We will conclude with a brief discussion on other aspects of theoretical modeling of organic electronic materials. Carrier transport in typical organic semiconductor, like rubrene or pentacene, is characterized by two regimes in terms of mobilities. At high temperatures, which normally include room conditions, there is a strong coupling between carriers and molecular vibrations that leads to polaron formation. The mobility is then described by Marcus theory, $[4,6]$ and the key quantities that can be calculated from first-principles [6] are the so-called transfer integrals and the reorganization energies. The former describe the electronic coupling between orbitals, and the latter relate to the effective barrier for carrier hopping due to charging-decharging of molecules during carrier hopping. At lower temperatures the coupling between carriers and phonons is weak and the transport can be described by the Miller-Abrahams model.[3,6] Once the key parameters are obtained from theory, Monte-Carlo simulations of hopping can describe charge and energy transfer in a larger scale, taking also into account spatial inhomogeneities and disorder.[7]

\section{Summary}

In summary, the theoretical modeling of organic electronic materials poses many types of challenges, as they normally span different scales in space and time. The availability of increasing computational power has allowed for an expanded role for first-principles quantum mechanical calculations to address several of these challenges, as we demonstrated with pertinent examples on the electronic, structural, and chemical properties of selected prototype organic materials.

\section{Acknowledgments}

We acknowledge support by the William A. and Nancy F. McMinn Endowment at Vanderbilt University, and by DOE Grant No DEFG0203ER46096. The calculations were performed at ORNL's Center for Computational Sciences.

\section{References}

1. C. D. Dimitrakopoulos, S. Purushothaman, J. Kymissis, A. Callegari, and J. M. Shaw, Science 283, (1999) 822.

2. M. Bendikov, F. Wudl, and D. F. Perepichka, Chem. Rev. 104, (2004) 4891.

3. J. L. Brédas, D. Beljonne, V. Coropceanu, and J. Cornil, Chem. Rev. 104, (2004) 4971.

4. M. E. Gershenson, V. Podzorov, and A. F. Morpurgo, Rev. Mod. Phys. 78, (2006) 973.

5. J. T. Mabeck and G. G. Malliaras, Adv. Funct. Mater. 384, (2006) 343 . 
6. V. Coropceanu, J. Cornil, D. A. Filho, Y. Olivier, R. Silbey, and J. L. Brédas, Chem. Rev. 107, (2007) 926.

7. A. B. Walker, A. Kambili, and S. J. Martin, J. Phys. Condens. Matt. 14, (2002) 9852.

8. J. P. Perdew and A. Zunger, Phys. Rev. B 23, (1981) 5048.

9. J. P. Perdew and Y. Wang, Phys. Rev. B 45, (1992) 13244.

10. D. Vanderbilt, Phys. Rev. B 41, (1990) 7892.

11. P. E. Blöchl, Phys. Rev. B 50, (1994) 17953.

12. G. Kresse and J. Furthmuller, Phys. Rev. B 54, (1996) 11169.

13. D. J. Chadi, and M. L. Cohen, Phys. Rev. B 8, (1973) 5747.

14. G. Mills, H. Jónsson, and G. K. Schenter, Surf. Sci. 324, (1995) 305 .

15. L. Tsetseris and S. T. Pantelides, Phys. Rev. Lett. 97, (2006) 116101.

16. L. Tsetseris, N. Kalfagiannis, S. Logothetidis, and S. T. Pantelides, Phys. Rev. Lett. 99, (2007) 125503.

17. R. Ruiz, D. Choudhary, B. Nickel, T. Toccoli, K. C. Chang, A. C. Mayer, P. Clancy, J. M. Blakely, R. L. Headrick, S. Iannotta, and G. G. Malliaras, Chem. Mater. 16, (2004) 4497.

18. R. B. Campbell, J. M. Robertson, and J. Trotter, Acta Crystallogr. 14, (1961) 705.

19. O. Jepsen and O. K. Andersen, Sol. Stat. Comm. 9, (1971) 1763.

20. E. A. Silinsh, V. A. Kolesnikov, I. J. Muzikante, and D. R. Balode, Phys. Stat. Sol. B 113, (1982) 379.

21. M. L. Tiago, J. E. Northrup, and S. G. Louie, Phys. Rev. B 67, (2003) 115212.

22. V. C. Sundar, J. Zaumseil, V. Podzorov, E. Menard, R.

L. Willett, T. Someya, M. E. Gerzhenson, and J. A. Rogers, Science 303, (2004) 1644.
23. O. D. Jurchescu, A. Meetsma, and T. T. Palstra, Acta Crystal. B 62, (2006) 330.

24. L. Tsetseris and S. T. Pantelides, unpublished.

25. J. E. Northrup and M. L. Chabinyc, Phys. Rev. B 68, (2003) 041202.

26. L. Tsetseris and S. T. Pantelides, Phys. Rev. B 75, (2007) 153202.

27. O. Mitrofanov, D. V. Lang, C. Kloc, J. M. Wikberg, T. Siegrist, W. Y. So, M. A. Sergent, and A. P. Ramirez, Phys. Rev. Lett. 97, (2006) 166601.

28. C. Krellner, S. Haas, C. Goldmann, K. P. Pernstich, D. J. Gundlach, and B. Batlogg, Phys. Rev. B 75, (2007) 245115.

29. D. V. Lang, X. Chi, T. Siegrist, A. M. Sergent, and A. P. Ramirez, Phys. Rev. Lett. 93, (2004) 086802.

30. L. Tsetseris and S. T. Pantelides, Phys. Rev. Lett. 97, (2006) 266805.

31. L. Tsetseris and S. T. Pantelides, Appl. Phys. Lett. 87, (2005) 233109.

32. L. Tsetseris and S. T. Pantelides, Mater. Sci. Eng. B (to be published).

33. B. Silvi and A. Savin, Nature 371, (1994) 683.

34. S. Yoo, J. J. Zhao, J. L. Wang, and X. C. Zeng, J. Am. Chem. Soc. 126, (2004) 13845.

35. L. Tsetseris, G. Hadjisavvas, and S. T. Pantelides, Phys. Rev. B 76, (2007) 045330.

36. G. de la Torre, P. Vázquez, F. Agulló and T. Torres, J. Mater. Chem. 8, (1998) 1671.

37. C. G. Claessens, U. Hahn, and T. Torres, Chem. Rec. 8, (2008) 75 .

38. J. Janczak and R. Kubiak, J. Alloys. Comp. 190, (1992) 121. 
39. T. Strenalyuk, S. Samdal, and H. V. Volden, J. Phys.

Chem. 112, (2008) 4853. 